\title{
Erratum to: The effect of Knudsen layers on rarefied cylindrical Couette gas flows
}

\author{
Nishanth Dongari - Robert W. Barber • \\ David R. Emerson • Stefan K. Stefanov • \\ Yonghao Zhang $\cdot$ Jason M. Reese
}

Published online: 14 March 2013

(C) Springer-Verlag Berlin Heidelberg 2013

\section{Erratum to: Microfluid Nanofluid (2013) 14:31-43 DOI 10.1007/s10404-012-1019-2}

The original publication of the article contains errors which need to be amended as mentioned below.

The text immediately after Eq. (8) should read as: "where $p(r)$ describes the probability a molecule will experience a collision while travelling a distance $r$."

The corrected version of Fig. $1 \mathrm{~b}$ is given here.

The corrected version of Eq. (19) is given below:

$R_{2}^{2}=r^{2}+\left(R^{+}\right)^{2}+2 r R^{+} \cos \left(\theta^{+}\right)$,

After Eq. (20), in the second line of the paragraph text should read as:

"Using half symmetry, it is sufficient to integrate $\theta^{+}$ from 0 to $\pi$."

The corrected versions of Eqs. (21) to (24) can be written as follows:

The online version of the original article can be found under doi:10.1007/s10404-012-1019-2.

N. Dongari $\cdot$ Y. Zhang $\cdot$ J. M. Reese

Department of Mechanical and Aerospace Engineering, University of Strathclyde, Glasgow G1 1XJ, UK

N. Dongari $(\bowtie) \cdot R$. W. Barber $\cdot$ D. R. Emerson STFC Daresbury Laboratory, Centre for Microfluidics and Microsystems Modelling, Warrington WA4 4AD, UK e-mail: nishanth.dongari@strath.ac.uk

\section{S. K. Stefanov}

Institute of Mechanics, Bulgarian Academy of Sciences,

Acad. G. Bonchev Str., Bl. 4, 1113 Sofia, Bulgaria
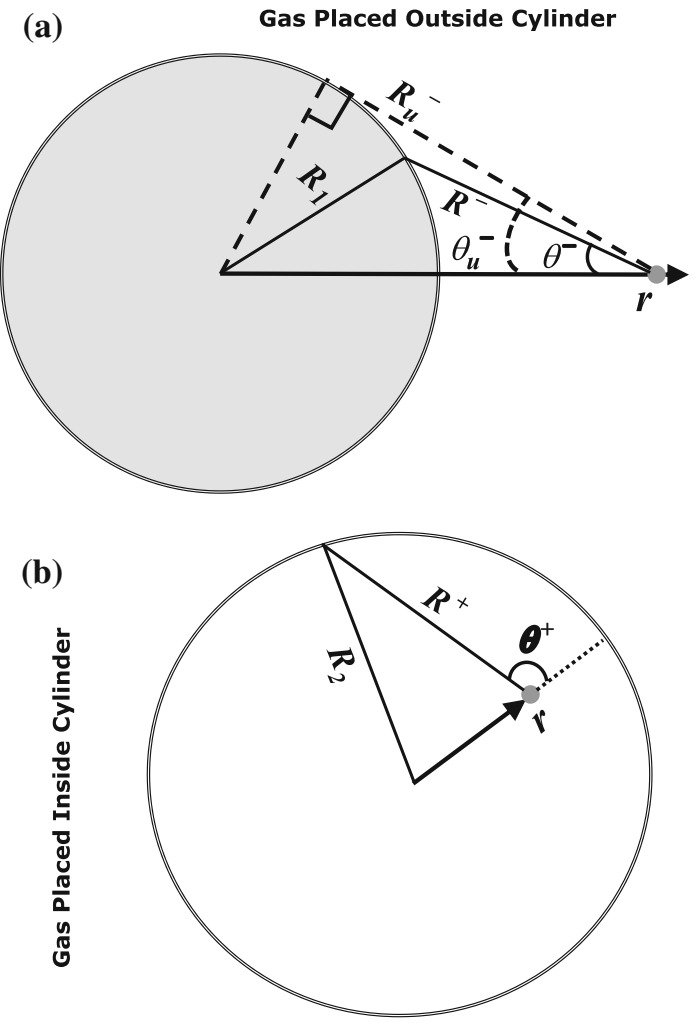

Fig. 1 a A gas molecule outside a solid cylinder and situated at a radial distance $r$ from the centre of the cylinder of radius $R_{1} . R^{-}$is the travelling distance limit for a molecule moving towards the cylinder surface, for a given zenith angle $\theta^{-}$. The largest travelling distance $R_{\mathrm{u}}^{-}$ is achieved for the zenith angle direction $\theta_{\mathrm{u}}^{-}$, above which the molecule by-passes the cylinder surface and travels into the bulk. b A gas molecule inside a cylindrical cavity of radius $R_{2}$, at a wall normal distance of $R_{2}-r$, where $r$ is the radial distance of the molecule from the centre of the cylinder. The molecule has a traveling distance of $R^{+}$to the wall for a traveling direction of $\theta^{+}$, where $\theta^{+}$is varied from 0 to $\pi$ 
$\lambda_{\mathrm{eff}(\mathrm{conc})}=\lambda\left[1-\frac{1}{\pi} \int_{0}^{\pi}\left(1+\frac{R^{+}\left(r, \theta^{+}\right)}{a}\right)^{(1-n)} \mathrm{d} \theta^{+}\right]$

$\beta_{(\mathrm{i})}=\frac{\lambda_{\mathrm{eff}(\mathrm{conc})}}{\lambda}=1-\frac{1}{\pi} \int_{0}^{\pi}\left(1+\frac{R^{+}\left(r, \theta^{+}\right)}{a}\right)^{(1-n)} \mathrm{d} \theta^{+}$,

$\lambda_{\mathrm{eff}}=\lambda_{\mathrm{eff}(\mathrm{conv})}\left(\frac{\theta_{u}^{-}}{\pi}\right)+\lambda_{\mathrm{eff}(\mathrm{conc})}\left[1-\left(\frac{\theta_{u}^{-}}{\pi}\right)\right]$

$\beta=\left(\frac{\theta_{u}^{-}}{\pi}\right)\left[1-\frac{1}{\theta_{u}^{-}} \int_{0}^{\theta_{u}^{-}}\left(1+\frac{R^{-}\left(r, \theta^{-}\right)}{a}\right)^{(1-n)} \mathrm{d} \theta^{-}\right]$

$+\left[1-\left(\frac{\theta_{u}^{-}}{\pi}\right)\right]\left[1-\frac{1}{\theta_{u}^{+}} \int_{0}^{\theta_{u}^{+}}\left(1+\frac{R^{+}\left(r, \theta^{+}\right)}{a}\right)^{(1-n)} \mathrm{d} \theta^{+}\right]$,
In Sect. 2.3, Line 10, the inline equation should be replaced by

$\left[1-\left(\theta_{u}^{-} / \pi\right)\right]$

In Sect. 3.1, line 4 in the first paragraph, the text Fig. 1 is replaced by Fig. 5 . 